
\title{
R Reserarch Suare \\ Real-time Visibility, Operational Insights and Actions with SAP Operational Process Intelligence, powered by HANA
}

Harsh Jegadeesan ( $\square$ harsh.jegadeesan@gmail.com )

SAP SE https://orcid.org/0000-0003-0448-0139

\section{Short Report}

Keywords: operational intelligence, $\mathrm{Al} / \mathrm{ML}$

Posted Date: October 26th, 2021

DOI: https://doi.org/10.21203/rs.3.rs-1016519/v1

License: (c) (1) This work is licensed under a Creative Commons Attribution 4.0 International License.

Read Full License 


\title{
Real-time Visibility, Operational Insights and Actions with SAP Operational Process Intelligence, powered by HANA
}

\author{
Dr. Harsh Jegadeesan, Thomas Volmering \\ Integration and Orchestration, HANA Platform
}

October 25, 2021

\begin{abstract}
Over the past decade, business operations in organizations are increasingly generating vast amounts of data. This steep increase in operational data - often known as big data - is a result of organizations capturing data at more fine-granular levels in business operations. Leveraging operational big data to improve business operations can create sustainable competitive advantage to organizations. In this paper, we argue that operational big data is "dead data" unless it is contextualized and made actionable for line-of-business users. Moreover, we explain how organizations can use SAP Operational Process Intelligence and the power of HANA to first, contextualize operational big data for real-time operational intelligence, second, provide line-of-business users visibility into business operations and business situations as they evolve and third, propose appropriate actions to respond to these business situations and help them reach business outcomes.
\end{abstract}

\section{Introduction}

Over the past decade, business operations in organizations are increasingly generating vast amounts of data. This steep increase in operational data - often known as big data - is a result of organizations capturing data at more finegranular levels in business operations. In addition to transactional data captured in traditional operational systems like ERP (enterprise resource planning) and CRM (customer relationship management) systems, organizations are also capturing machine data and clickstream data e.g. web server logs in online retail stores, call data records in telecom, GPS location data in transportation and logistics etc. This high-volume of operational data captured is widely diverse - diverse in sources (databases, server logs, sensors, scanners), diverse in structures (structured, un-structured and semi-structured), diverse in speed and frequency at which data is generated (streaming data, transactional data, master data). 
All of this diverse operational big data, if leveraged properly, can be a treasure trove of information to learn about customer behavior, operational bottlenecks and weak spots and help in constantly improving business operations. In addition to these post-facto learnings, organizations can achieve real-time operational intelligence when streaming data from new sources is combined with historical data from traditional business systems in real-time.

Real-time Operational intelligence empowers front-line employees with contextualized process visibility, intelligent insights and enables them to take the right actions based on emerging business situations so as to reach desirable business outcomes.

In this paper, we describe a way to contextualize large volumes of operational big data and make it actionable for line-of-business users. Thereby enable them to sense and respond to threats and opportunities in real-time. The rest of this paper is structured as follows: in section 2, we argue that operational big data is "dead data" - data that is no longer relevant or useful - unless it is contextualized and made actionable in real-time to front-line employees of the organization. We propose to contextualize operational big data around endto-end business processes that represent business operations. In section 3, we present our approach of providing real-time operational intelligence for line-ofbusiness users using SAP Operational Process Intelligence and the power of HANA. In section 4, we present outlook and future work and in section 5 , we provide conclusions.

\section{Contextualizing and Making Operational Big Data Actionable}

Massive volumes of operational big data that results from fine-granular data capture in business operations can be useless for making operational decisions, unless it is contextualized in real-time for front-line employees. Therefore, the first step towards real-time operational intelligence is to contextualize these vast amounts of operational big data for employees such as operational managers, service agents and customer relationship executives. Contextualization helps them understand and relate to it and make use of it to achieve business outcomes. These massive amounts of operational data will be "dead data" if it is not contextualized and actionable in a timely fashion for line-of-business users.

From our perspective end-to-end business processes provide the right context for operational big data. Business users typically understand business operations as end-to-end business processes. In addition, research strongly suggests that companies can enhance the performance of their daily business operations by taking a "process view" of the organization. Business process and value-stream orientation has proved to reduce interdepartmental conflicts, improve crossfunctional collaboration, and significantly increase customer focus. ${ }^{1}$

\footnotetext{
${ }^{1} \mathrm{KP}$ McCormack and, WC Johnson, "Business Process Orientation: Gaining the e-business
} 


\subsection{Contextualizing Operational Big Data into Big Pro- cesses}

In every organization, end-to-end business processes support business operations and in-turn the organization's business model. Organizations have to move away from the splintered and siloed views of business to "big-process thinking". 2 Big Processes are end-to-end business scenarios focused on delivering value to customers. ${ }^{3}$ Departments within companies can no longer work in isolation; instead employees from several departments must collaborate to deliver value to customers and stakeholders.

From a technology perspective, these big processes usually span multiple technical systems - packaged applications, enterprise service bus as well as operations support systems (web servers, manufacturing systems, sensors etc.). From an organizational perspective, big processes span several departments as many people collaborate to reach common goals.

All the operational data generated by these systems can be contextualized around this big process easily. Line-of-business users see big processes as endto-end processes with clear goals and in well-defined phases and milestones (see figure 1). Goals define business outcomes from the perspective of the organization. Milestones are intermediate checkpoints to ensure that that goal is reachable. Phases represent the high-level value stream from the perspective of business. Success is measured based on real-time metrics and key performance indicators. Business Situations are undesired situations or contingencies in business operations that need to be sensed in advance and responded to by taking appropriate actions. Actions remedy business situations and in addition, help line-of-business users to reach milestones and goals.

In summary, if streaming business events is correlated to business data in transactional systems and contextualized into end-to-end processes then line-ofbusiness users can relate to it far easily and can leverage this to reach business outcomes.

\subsection{Example: Managing Logistics Hub Operations}

Consider the example of a cross-docking logistics hub operated by an express logistics service provider. A cross-docking hub handles on an average between 250,000 and 400,000 shipments in a period of five to six hours every night. Shipments arrive in inbound planes that are automatically guided to a parking position by the air traffic controller. Pallets (also known as unit-loading devices - ULDs) that contain the shipments are unloaded in the apron by high-loaders.

\footnotetext{
competitive advantage," 2001.

${ }^{2}$ Clay Richardson, Craig Le Clair, Alex Cullen, and Julian Keenan, "Embrace Five Disruptive Trends That Will Reshape BPM Excellence," Forrester Research Inc.2012

${ }^{3}$ Harshavardhan Jegadeesan, "Tame BIG Processes with SAP Operational Process Intelligence, powered by SAP HANA - Part 1," SAP Community Network, blog, March 17, 2013.
} 


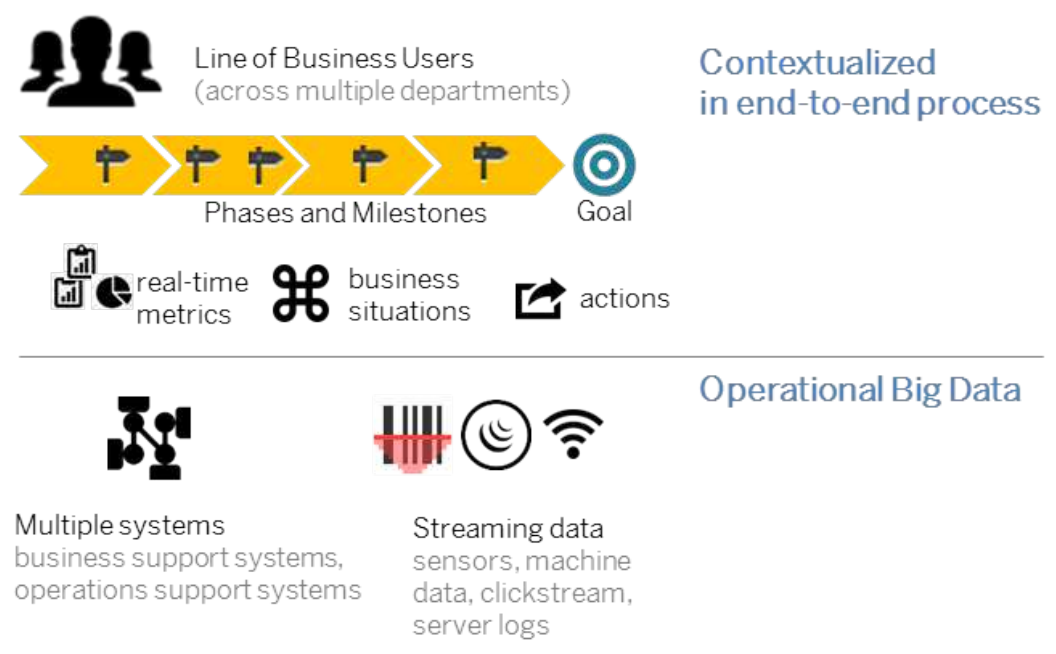

Figure 1: Contextualizing operational big data into end-to-end processes

Workers move the pallets to the warehouse in tugs and trolleys, open them up, and put all shipments on long conveyer belts for sorting. Shipments are repaired if they are damaged or go through customs clearance, if needed, and are loaded onto an outbound plane or a road container for the next destination. All the departments and teams work toward a common goal of ensuring that all shipments that arrive in the hub are sorted and transited successfully every night. People involved in the hub operations - the shift manager, warehouse agent, and supervisors - relate to the end-to-end process of shipment movement across the warehouse. They see this process as a value stream of phases and milestones such as plane arrival, unloading, sorting in the warehouse, and loading on the outbound plane (see figure 2).

Multiple IT systems are involved, such as the freight management system, aviation control systems, and workforce allocation systems. The operational systems (sensors, bar code readers, air traffic control systems) generate close to 18 million events. For the people in the hub, all this operational big data ( 18 million events) need to be correlated to shipments and contextualized around the shipment movement process in the hub.

The goal of the hub operations manager is to ensure that every shipment that enters his cross-docking hub is sent out the same day and is not stored. For any reason, if a shipment is not sent out the same day, it is delayed and the logistics service provider loses money. Therefore Average Number of Retained Shipments (needs to be close to zero) is a critical metric that quantifies the goal of the hub operations manager.

By contextualizing and correlating the real-time events with the data in the freight management transactional system, the hub manager is presented with the number of shipments in different phases across the value stream as well as 
Figure 2: Example of Logistics Hub Operations

high-level traffic lighting to show which shipments are in danger of delaying outbound planes. In addition, he or she sees real-time metrics, such as time taken for loading or unloading a plane, number of shipments in the customs check area etc.

The goal of the hub operations manager is to ensure that every shipment that enters his cross-docking hub is sent out the same day and is not stored. For any reason, if a shipment is not sent out the same day, it is delayed and the logistics service provider loses money. Therefore Average Number of Retained Shipments (needs to be close to zero) is a critical metric that quantifies the goal of the hub operations manager.

\subsection{For Operational Intelligence, Real-time is the Right time}

Several operational decisions are still made based on regular reports produced daily, weekly or monthly. As a result, business situations that emerge during daily operations are rarely sensed and responded to in time. This can have adverse business impact such as loss of customer trust, financial liabilities etc. Latency in acquiring data from multiple operational systems, latency in analyzing this data from these many "keyholes" and "creating a big picture view" as well as latency in decision making results in many missed opportunities in responding to threats and seizing opportunities.

For example, in the logistics hub, the hub operations manager could face several contingencies (we refer to contingencies as business situations) in the course of daily operations. For example, flights may not take off due to technical snags or due to bad weather. A particular destination may have high-load of shipments in terms of volumetric weight or oversize shipments piling up in the loading dock. In order to manage these evolving business situations, the hub operations manager should have the capability to sense these business situations well in advance before they snowball into big problems. Therefore, contextualized insights help the hub operations managers only if it is in real-time. 
Post-facto analysis of operational big data using data warehouses, business intelligence and reporting software is useful to identify trends and learn from the past. However operational managers need real-time operational intelligence to manage present day's operations, sense business situations as they happen and respond to them with appropriate actions before they escalate into a crisis.

\subsection{Making Operational Big Data Actionable}

While line-of-business users value real-time visibility into business operations and evolving business situations, they value taking the right actions to reach goals and achieve business outcomes more. The notion of goals, progressing through phases and milestones in the value-stream to reach goals is a very important notion to make operational big data actionable. When line-of-business users sense evolving business situations that need attention, they take appropriate actions to mitigate these situations and still reach milestones and goals.

For example, if the hub operations manager gets to know the volume of shipments to a particular destination is too high, he can respond by taking corrective actions such as assigning a bigger plane or booking extra capacity in a flight owned by a commercial airline for that destination.

\section{Operational Process Intelligence and the HANA effect}

SAP Operational Process Intelligence (OPInt) is a new breed of real-time twotier application built natively inside the SAP HANA platform. OPInt provides real-time operational intelligence to organizations by:

- correlating and contextualizing massive volumes of operational big data from multiple diverse sources (SAP and non-SAP) into end-to-end processes.

- representing end-to-end process value-stream as phases and milestones thereby hiding all the technical details from line-of-business users.

- providing real-time metrics and key performance indicators.

- detecting business situations such as KPI violations and alerting responsible business users

- providing capability to business users to collaboratively solve problems before they turn into escalations

- proposing appropriate actions to business users to respond to business situations 


\subsection{Business scenario Contextualizes Operational Big Data}

The leading artifact in SAP Operational Process Intelligence is the business scenario (see figure 3).

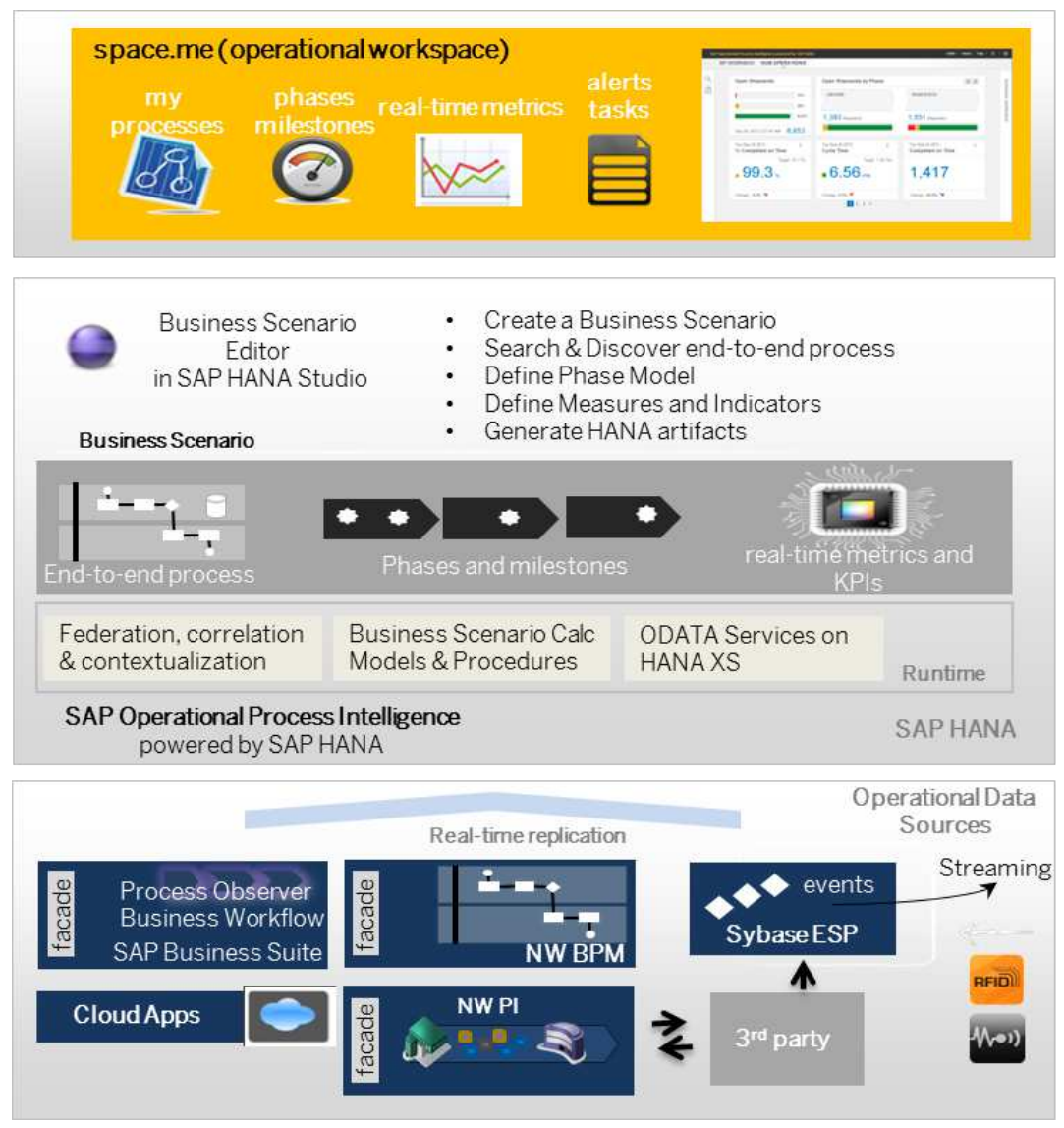

Figure 3: Business scenario in SAP Operational Process Intelligence

Business scenario is a native HANA repository artifact that is configured by a solution expert who understands the business operations using the business scenario editor. The business scenario editor can be installed on top of the SAP HANA studio. The business scenario helps to contextualize the vast amounts of operational big data in a form that line-of-business users understand and relate to. A business scenario has three main components:

First, the business scenario captures the end-to-end flow of a big process. Most importantly, the end-to-end flows need not be automated, or even be in the control of one single system. A solution expert can assemble a big process from different process fragments running in multiple SAP and non-SAP software systems and operational data providers. SAP Operational Process Intelligence provides ready support to discover process fragments from business workflows and from transactions from SAP Business Suite software, the SAP NetWeaver Business Process Management component, the SAP NetWeaver Process Inte- 
gration offering, and third-party software.

Second, the business scenario captures the high-level phases and milestones. Phases abstract the technical view and provide a value-stream view to lineof-business users. Phases are configured on the basis of process events that originate from the end-to-end flow configured in the previous step.

Third, the business scenario captures key metrics and process indicators such as cycle times and durations.

After configuring the business scenario, the solution expert does not need to code the artifacts for SAP HANA. When the business scenario is deployed, SAP Operational Process Intelligence generates optimized native run-time artifacts for SAP HANA. These include calculation views, correlation procedures, and OData services (Open Data Protocol) that are used to expose operational workspaces to line-of-business users in Space.me, a HTML5-based responsive interface served out the HANA extended application services (HANA XS).

\subsection{Data Acquisition, Correlation and Contextualization in Real-time}

Operational data in the form of process events and process data from different underlying systems (SAP Business Suite, NetWeaver Business Process Management, NetWeaver Process Integration, 3rd party application) involved in a business scenario are replicated into HANA in real-time. OPInt uses real-time data provisioning technologies such as SLT (System Landscape Transformation) for transactional systems. Streaming events are pushed into HANA using the event streaming technology - Sybase $\AA$ ESP (Event Stream Processor). Realtime availability of relevant operational data reduces the data acquisition latency. If certain operational systems such as the Business Suite or NetWeaver Process Orchestration run on the same HANA instance, then there is no need for replication of operational data.

The OPInt runtime uses the power of HANA to correlate and contextualize operational data into end-to-end processes. For example, in the logistics hub operations scenario, 18 million process events are correlated and contextualized into shipments across different phases in the hub (see figure 4).

\subsection{Detecting Business Situations using Business Rules and Predictions}

SAP Operational Process Intelligence can help line-of-business users sense business situations and respond to these situations as they evolve in real-time. Business situations represent undesired situations or contingencies in business operations that needs an appropriate response. Currently, there are three ways to detect business situations: 


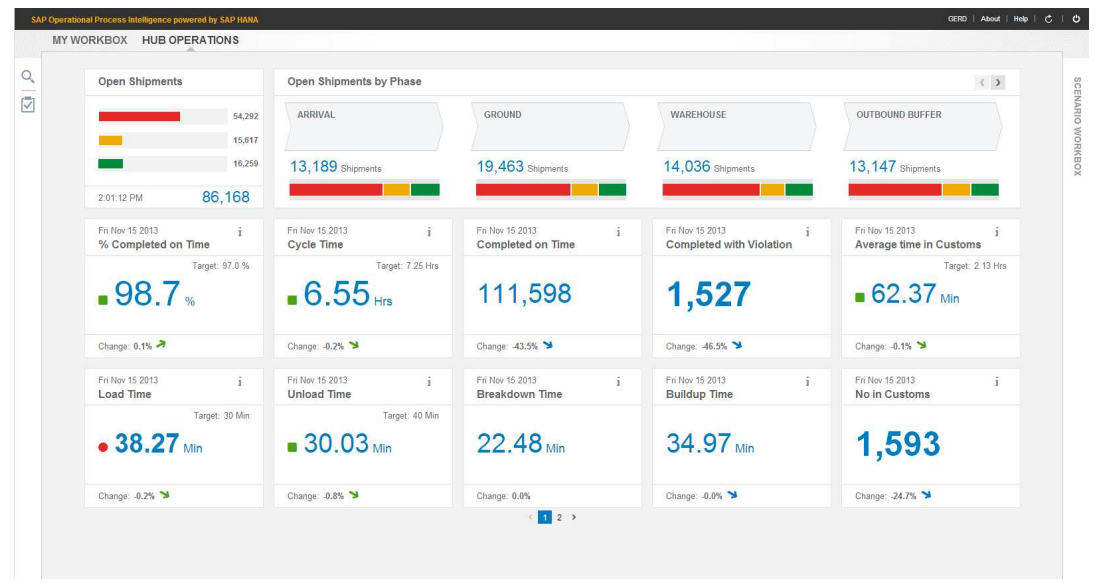

Figure 4: Business scenario: Logistics Hub Operations

First, when real-time metrics cross certain thresholds or key performance indicators are violated, a business situation occurs. For example, a real-time metric such as Loading Time in the hub operations scenario crossing a threshold leads to a business situation that needs attention and action. When such business situations occur, the appropriate business users are alerted.

Second, business rules can be defined natively inside HANA using decision tables that help to detect evolving business situations based on events, context and pre-conditions.

Third, predictive algorithms such as time series algorithms can be used to find out instances that may violate cycle time durations in the future or clustering algorithms may detect critical instances clustering around certain dimensions. This is a business situation that needs to be resolved.

Business users can collaboratively work on resolving these business situations and restore normalcy. Responding to business situations as they evolve is a significant differentiator for a real-time operational intelligence offering like SAP Operational Process Intelligence. Such a capability clearly differentiates it from traditional operational BI (business intelligence) tools.

\subsection{Support for Next-Best Actions}

At present, OPInt supports actions that can be configured by a solution expert in a business scenario. Actions allow business users to navigation to backend and third-party systems to perform certain operations such as release a purchase order, schedule a delivery, or re-plan workforce roster.

In the next release, OPInt will propose next-best actions to business users involved in daily business operations to mitigate evolving business situations. For example, when the Loading Time (time taken to load shipments into an onward 
flight) crosses the threshold, the hub operations manager is alerted and is proposed to take an action - Assign a T4 loading squad to the loading dock to speed loading. In another example, business rules can evaluate volumetric weight of shipments to different destinations and can automatically propose Change of Plane (from ATR -42 to Airbus A300) if the volumetric weight is higher.

From a technical standpoint, actions will be tightly integrated into the HANA platform programming model and will be semantically part of the core data services (CDS) - the data definition language of the HANA platform. Actions can be realized by HANA procedures or server-side JavaScript functions. Actions could also be realized as application workflows built inside HANA (see section 4)

\section{Outlook and Roadmap}

In the next releases, we will focus on the following high-level themes:

Next-Best Action Proposals Next-best Actions will be proposed based on occurrence of specific events or business situations, certain pre-conditions being met. Going further, we plan to use statistical mechanisms such as collaborative filtering (with explicit feedback) to validate the accuracy of next-best action proposals and refine the proposal constantly.

Workflows for Actions Certain actions will need to trigger workflows in order to accomplish a result. For example, in the logistics hub Book Commercial Airline Capacity action may trigger an approval workflow to book capacity in commercial airlines for a particular route. In order to support such use-cases, we are building application workflows as native HANA artifacts.

Enhancing HANA Rule Framework for supporting automatic actions At present, decision tables in HANA are used to automate operational decisions. Moreover, decision tables are used to define rules to determine business situations. As a next step, we will enhance the HANA Rules framework to re-use the vocabulary defined with core data services (CDS). This will enable us to leverage actions in decision tables natively. With this capability, we will be able to define event-condition-action rules that can trigger automatic actions on certain business conditions.

Intelligent Insights We plan to provide intelligent insights for business users to zero-in on problems areas faster. For example, highlight problem clusters, operational bottlenecks and weak spots and help business users to collaboratively apply these insights to solve operational problems.

Real-time optimizations We will support real-time optimization of resources - human, machine, financial - in business operations. For example, in the logistics hub, depending on real-time visibility into the capacity utilization 
of planes, the hub operations manager can merge flights into a single plane or re-route the shipment via a different route.

\section{Conclusions}

Operational big data is reality for several organizations. In this paper, we argue using an example from a logistics hub that:

1. Operational big data is "dead data" unless it is contextualized for lineof-business users. Moreover, we argued why end-to-end processes that represent business operations and the value-stream is the right way to contextualize operational big data

2. Real-time visibility into operations and evolving business situations is the key. Post-facto analysis may be complimentary and may help to learn from the past, however front-lines of business appreciate capabilities to sense and respond to threats and opportunities in real-time

3. Real-time visibility is the first step to make operational big data actionable. While the former is good, the latter is more valuable for front-lines of business.

Given this, we explained how SAP Operational Process Intelligence leverages the power of the HANA in-memory platform to help organizations gain real-time operational intelligence and thereby sustainable competitive advantage. 